\title{
Development of the Data Acquisition and Processing System for a Pulsed 2-Micron Coherent Doppler Lidar System
}

\author{
Jeffrey Y. Beyon ${ }^{* a}$, Grady J. Koch ${ }^{\mathrm{b}}$, and Michael J. Kavaya ${ }^{\mathrm{b}}$ \\ ${ }^{a}$ NASA Langley Research Center, MS 488, Hampton, VA 23681, USA; \\ ${ }^{b}$ NASA Langley Research Center, MS 468, Hampton, VA 23681, USA
}

\begin{abstract}
A general overview of the development of a data acquisition and processing system is presented for a pulsed, 2-micron coherent Doppler Lidar system located in NASA Langley Research Center in Hampton, Virginia, USA. It is a comprehensive system that performs high-speed data acquisition, analysis, and data display both in real time and offline. The first flight missions are scheduled for the summer of 2010 as part of the NASA Genesis and Rapid Intensification Processes (GRIP) campaign for the study of hurricanes. The system as well as the control software is reviewed and its requirements and unique features are discussed.
\end{abstract}

Keywords: Coherent lidar, GRIP, wind profile, DAWN AIR

\section{INTRODUCTION}

The project DAWN (Douppler Aerosol WiNd) is to study wind profiles using high-pulsed energy, 2 micron, solid-state coherent Doppler laser, which was developed at NASA Langley Research Center in Hampton, Virginia, USA. While the complete lidar system contains a laser, optics, telescope, and electronics, this paper focuses on the data acquisition system and its real-time software. We will present the aspects of design and performance of the data acquisition system and the software.

The system is called DAPDS (Data Acquisition, Processing, and Display System), which utilizes the cPCI architecture to control different subsystems that reside in it. The software running DAPDS is called DAPS (Data Acquisition and Processing Software), which is written in ANSI C. It is customized to perform high-speed data acquisition and analysis while controlling laser electronics and an INS/GPS unit.

This paper also presents the experiments conducted during the NASA Genesis and Rapid Intensification Processes (GRIP) during August - September, 2010. The system flew on a DC-8 flight laboratory through some hurricanes such as Earl an Gaston in late August and early September. Related photos and observations are presented in the latter section of this paper.

\section{HIGH-SPEED DATA ACQUISITION SYSTEM AND NASA MISSION GRIP}

The mission GRIP that stands for the Genesis and Rapid Intensification Processes is to investigate the birth and the development of tropical storms and hurricanes. Using the DC-8, the WB-57, and the Global Hawk Unmanned Airborne System (UAS), NASA's engineers and scientists observe and collect data of storms and hurricanes during August 15 September 30, 2010. The bases of each aircraft are Ft. Lauderdale, FL, Houston, TX, and NASA Dryden Research Facility, CA, respectively.

The project DAWN is a prelude to a space-born wind profile mission using a 2 micron, pulsed Doppler lidar system, and participated in the NASA GRIP hurricane campaign to test the airborne system. The laser optical bench and system are housed in a temperature controlled canister, which was located in the cargo area of the DC-8.

*Jeffrey.Y.Beyon@nasa.gov; phone 1757 864-4249; fax 1757 864-7944; www.nasa.gov 
The data acquisition, processing, and display system (DAPDS) and the laser control electronics were placed in the cabin area, where two or three operators worked on the system simultaneously. The chillers were also located in the cabin area behind the DAPDS and the laser control electronics. There were numerous project teams onboard and the payload is shown in Figure 1.

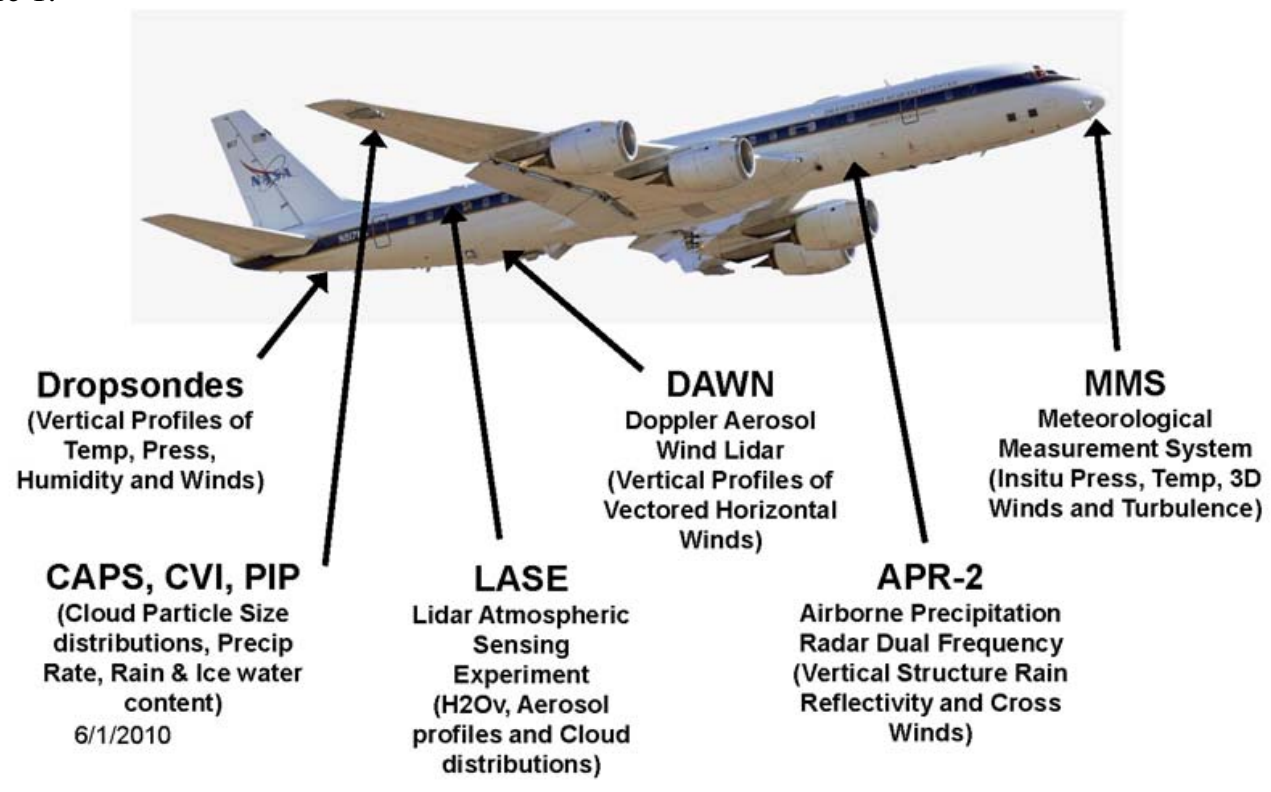

Fig. 1. GRIP DC-8 Payload

The DC-8 payload was integrated at NASA Dryden Operational Facilities in Palmdale, CA, USA in July. A shakedown flight and an engineering flight were administered in early August, 2010, followed by a ferry flight to Ft. Lauderdale, FL, USA on August 13, 2010. The first GRIP mission flight began on August 17, 2010, and the first flight through the hurricane Earl was on August 29, 2010. The DC-8 left for St. Croix, VI, USA on August 28, 2010, and stationed there overnight to fly through Earl the following day. After one day of exploration of Earl, the DC-8 was diverted to Ft. Lauderdale due to the inclement weather due to Earl. The hurricane Earl became category 3 on early August 30, and category 4 later that day. In order to entertain the project objectives of each science team, different types of maneuvers at a variety of altitudes were commanded.

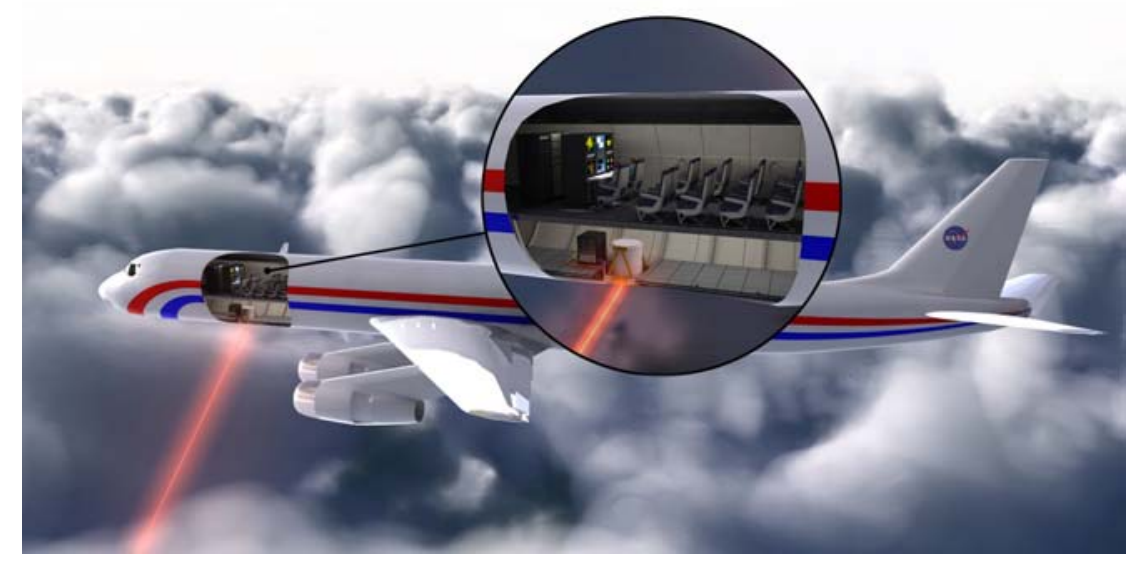

Fig. 2. DAWN AIR on the DC-8

The DAPDS consists of four subsystems: DSP subsystem (DSPSS), Digitizer subsystem (DSS), INS/GPS subsystem (IGSS), and Scanner subsystem (SCSS). Figure 3 depicts the DAPDS where the IGSS and the SCSS communicate with 
the data processing program via serial ports, the DSS via the backplane of the CPCI using the TTL trigger signal from the laser optics system, and the DSPSS via the backplane of the cPCI via bus mastering. The DSPSS consists of 8 DSP processors, which communicate with each other in order to process a block of data simultaneously.

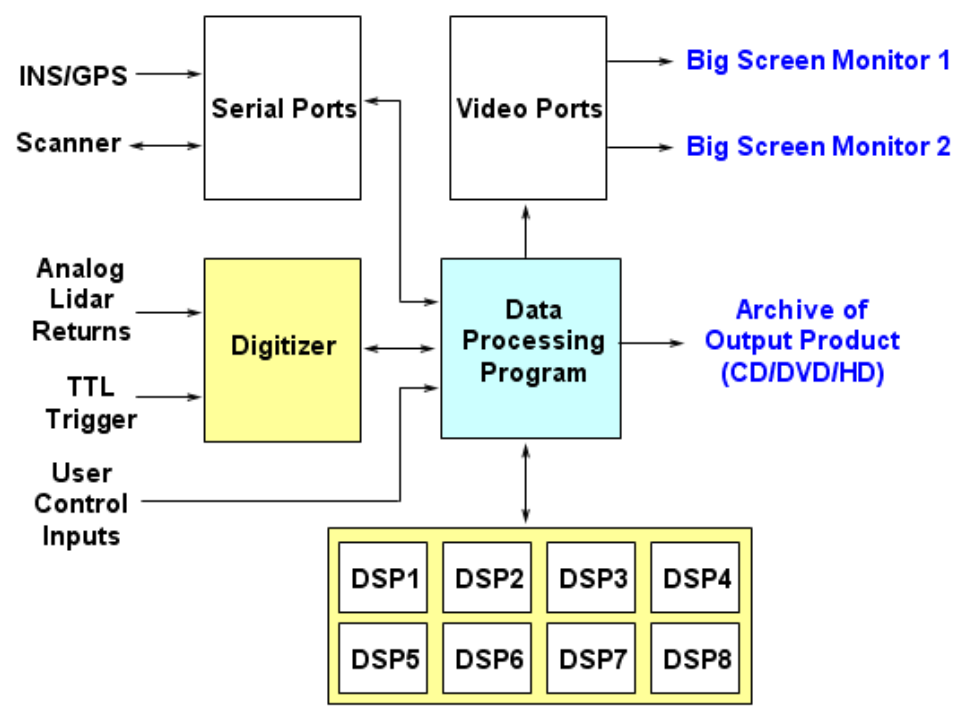

Fig. 3. Anatomy of the DAPDS

The overall system description includes (1) $500 \mathrm{MHz}$ sampling rate at 10-bit resolution, (2) $10 \mathrm{~Hz}$ real-time data acquisition and processing rate using an external TTL pulse, (3) control of scanner, (4) archive of lidar backscatter return data, (5) real-time wind profile parameter estimation and its display, and (6) a user-friendly input control window.

\section{SUBSYSTEMS OF THE DAPDS}

The DAPDS is a 6U 8-slot Compact PCI chassis by Kontron, housing a digitizer, a DSP module, a serial port module, and a video port module. The CPU runs XP-Pro at Intel $1.8 \mathrm{GHz}$ clock speed with $400 \mathrm{MHz}, 2 \mathrm{~GB}$ SDRAM. There are four subsystems in the DAPDS. Figure 4 shows them and their communication methods graphically.

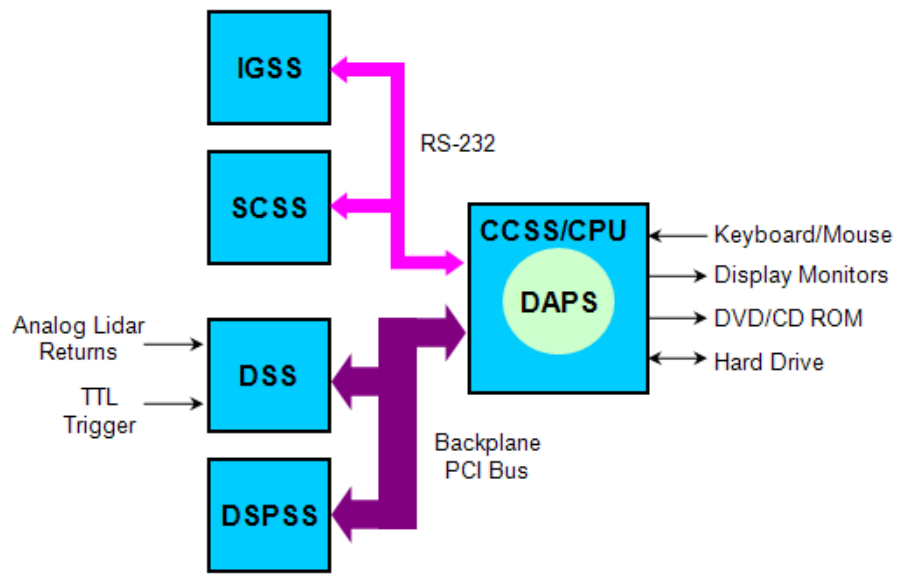

Fig. 4. Anatomy of the DAPDS

The IGSS is the C-MIGITS by Systron Donner for INS/GPS data. The SCSS is the scanner subsystem, which controls the scanner. Both the IGSS and the SCSS communicate with Data Acquisition and Processing Software (DAPS) via RS- 
232. The DSS is a quad-channel 10-bit resolution digitizer with $2-8 \mathrm{GHz}$ sampling rate with 256-1024 $\mathrm{K}$ samples. The last DSPSS is a BittWare TigerSHARC DSP module with two clusters of four DSPs. Each DSP runs at $600 \mathrm{MHz}$ with 1.67 ns Instruction Rate and 3.6 GFLOPS for 32-bit floating per DSP. It has native support for 32-bit floating point operations with 24 Mbit on-chip RAM per DSP.

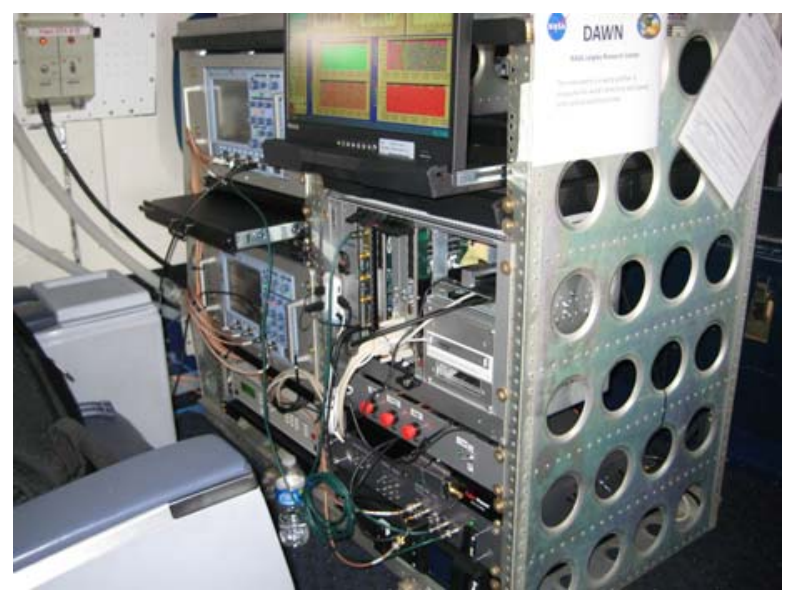

Fig. 5. The DAPDS and the laser electronics system in the DC-8 during the mission GRIP

During the mission GRIP, the DAPDS scanned five look directions, which were -45 degrees, -22.5 degrees, 0 degree, 22.5 degrees, and 45 degrees azimuth in reference to the nose of the aircraft ( 0 degree azimuth) and 60 degrees elevation (30 degrees from Nadir). At each line of sight (LOS), the system controlled the laser electronics so that twenty lidar pulses were emitted at $10 \mathrm{~Hz}$ rate, and acquired their backscatter signals while processing the data using the IGSS. The system was optimized for 512-point FFT processing with 50\% overlapped range bins for real-time processing. 55,000 samples were acquired per each lidar pulse, resulting 209 FFTs to process in $100 \mathrm{~ms}$. It also communicated with the IGSS at $10 \mathrm{~Hz}$ in order to acquire the INS/GPS data for each lidar pulse. DAPS controlled the laser electronics to move the scanner, acquire and process data, and display the results in the main window.

\section{DATA ACQUISITION AND PROCESSING SOFTWARE - DAPS}
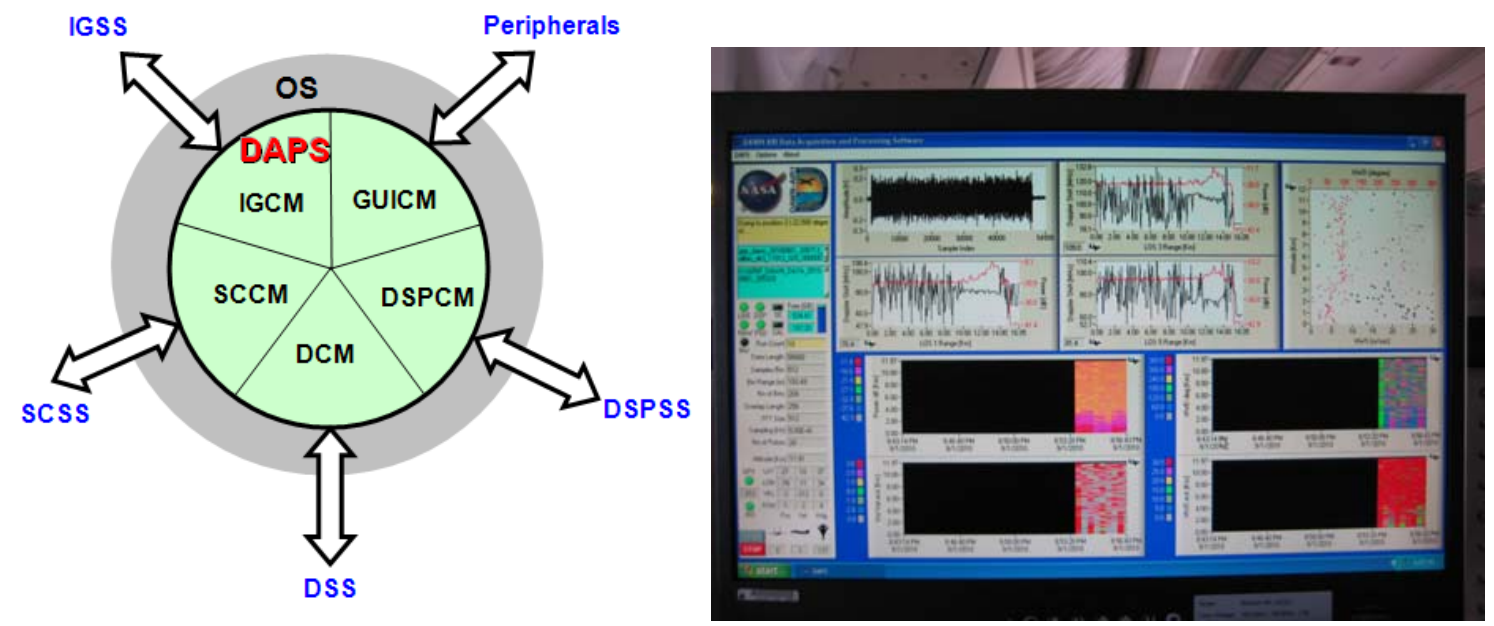

Fig. 6. Anatomy of DAPS and live action during the mission GRIP in the DC-8 
The real-time software called DAPS was written in ANSI C with the serial and the GUI drivers from National Instruments. Figure 6 shows the architecture of DAPS graphically and the picture of DAPS working during the mission GRIP in the air. DAPS runs in XP environment with five control modules: INS/GPS control module (IGCM), GUI control module (GUICM), DSP control module (DSPCM), digitizer control module (DCM), and scanner control module (SCCM). Each control module controls the sub system as implied by its name. The control modules were written in multithreads while data share was carefully controlled. Memory management and data transfer were carefully controlled in order to optimize the high-speed data processing and maintain high throughput.

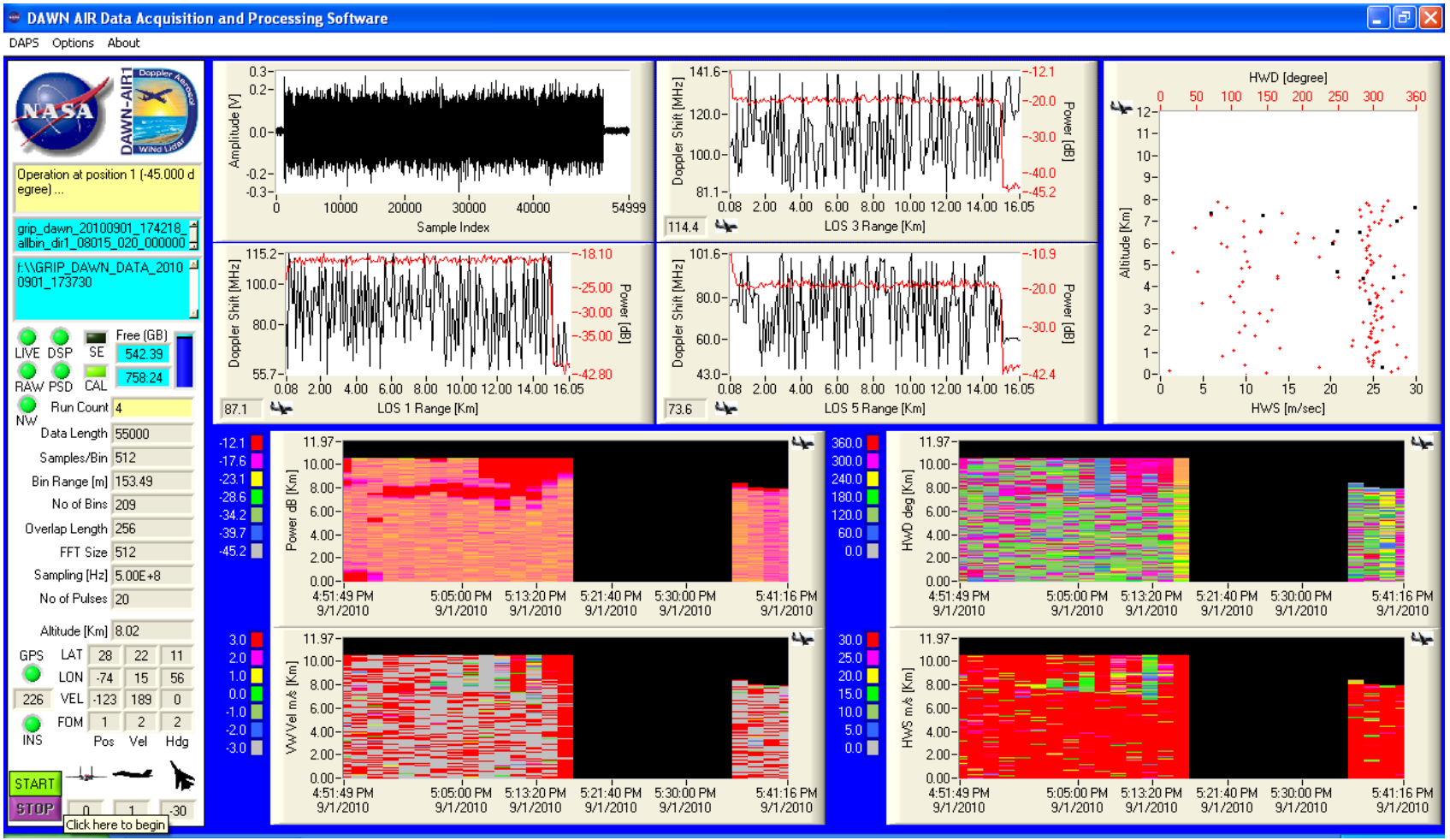

Fig. 7. A screenshot of DAPS in live action

\section{CONCLUSION}

The design of real-time data acquisition and analysis system for a coherent, 2-micron, pulsed wind lidar system for a project DAWN is introduced. The system is designed at NASA Langley Research Center, and is a preliminary showcase for a future space-born wind lidar mission. The latest participation in the NASA GRIP hurricane mission showed a promising results of airborne wind profiling. The detailed discussion of DAPS functionalities and wind parameter estimation algorithms are in the works for future papers. The real-time execution of DAPS along with the laser optics and electronics shows the advantage of coherent Doppler lidar system over direct detection lidar systems.

\section{ACKNOWLEDGMENT}

This work was supported by the NASA Instrument Incubator Program, the NASA Laser Risk Reduction Program, and the NASA Airborne Technology Transition program. 


\section{BIBLIOGRAPHY}

[1] J. Y. Beyon and G. J. Koch, "Novel Nonlinear Adaptive Doppler Shift Estimation Technique (NADSET) for the Coherent Doppler Lidar System VALIDAR,” in Proc. of the Defense and Security Symposium 2006 (62361), Orlando, FL, April, 2006.

[2] J. Y. Beyon and G. J. Koch, "Resolution Study of Wind Parameter Estimates by a Coherent Doppler Lidar System,” in Proc. of the Defense and Security Symposium 2006 (6214-3), Orlando, FL, April, 2006.

[3] J. Y. Beyon and G. J. Koch, "Wind Profiling by a Coherent Doppler Lidar System VALIDAR with a Subspace Decomposition Approach," in Proc. of the Defense and Security Symposium 2006 (6236-5), Orlando, FL, April, 2006.

[4] J. Y. Beyon, G. J. Koch, and Z. Li "Noise Normalization and Windowing Functions for VALIDAR in Wind Parameter Estimation," in Proc. of the Defense and Security Symposium 2006 (6214-4), Orlando, FL, April, 2006.

[5] J. Y. Beyon and G. J. Koch, "Novel nonlinear adaptive Doppler-shift estimation technique for the coherent Doppler validation lidar,” Optical Engineering, Vol. 46, No. 1, pp. 016002-1 - 016002-10, January 2007.

[6] J. Y. Beyon, G. J. Koch, and S. Ismail "Signal processing techniques for heterodyne differential absorption lidar,” in Proc. of the Defense and Security Symposium 2007 (6567-53), Orlando, FL, April, 2007.

[7] G.J. Koch, J.Y. Beyon, B.W. Barnes, M. Petros, J. Yu, F. Amzajerdian, M.J. Kavaya, and U.N. Singh, "HighEnergy 2- $\mu m$ Doppler Lidar for Wind Measurements," Optical Engineering, Vol. 46(11), pp. 116201-1 116201-14, November, 2007.

[8] J. Y. Beyon, G. J. Koch, M. J. Kavaya, and M. Sahota, "Comparison of theoretical and empirical statistics of wind measurements with validation lidar (VALIDAR)," in Proc. of the Defense and Security Symposium 2008(6968-59), Orlando, FL, March, 2008.

[9] B. Demoz, "The Howard University Beltsville Research Campus: Highlights from the Recent Wind Lidar and Water Experiemnts,” Wind Lidar Working Group Meeting, June, 2009.

[10] G. J. Koch, "Ground-Based Testing of the DAWN 2-Micron Doppler Lidar and Comparison with Other Sensors,” Wind Lidar Working Group Meeting, June, 2009.

[11] K. Vermeesch, G. Koch, B. Gentry, T. Bacha, H. Chen, and B. Demoz, "Comparisons of Ground-Based, Radiosonde, and Aircraft Wind Measurements at the Howard University Beltsville Research Site,” Wind Lidar Working Group Meeting, June, 2009. 